\title{
The ZIF-8 Based on Poly Microspheres as A Novel Adsorbent for Removing Organic Contaminants from Wastewater
}

\author{
Juan DAI ${ }^{1, a}$, Yan-Xue $\mathrm{LIU}^{2, \mathrm{a}}$, Shang-Zhen XIAO ${ }^{3, \mathrm{a}}$, Jing $\mathrm{LIU}^{4, \mathrm{a}}$, Jing $\mathrm{HE}^{5, \mathrm{a}}$, \\ Jian-Du LEI ${ }^{6, b}$ and Lu-Ying WANG ${ }^{a,{ }^{*}}$ \\ a Beijing Key Laboratory of Lignocellulosic Chemistry, Beijing Forestry University, Beijing, 100083, \\ China \\ ${ }^{\mathrm{b}}$ MOE Engineering Research Center of Forestry Biomass Materials and Bioenergy, Beijing Forestry \\ University, Beijing, 100083, China \\ 1'dai0520@bjfu.edu.cn (J. Dai), ${ }^{2} 835945592 @ q q . c o m\left(Y\right.$. Liu), ${ }^{3} 1426044125 @ q q . c o m$ (S. \\ Xiao), ${ }^{4}$ liujing@bjfu.edu.cn (J. Liu), ${ }^{5}$ hejing2008@sina.com (J. He), ${ }^{6}$ ljd2012@bjfu.edu.cn (J. Lei) \\ Corresponding author: wangly@bjfu.edu.cn (L. Wang)
}

Keywords: ZIF-8, poly microspheres, adsorption, Malachite Green

\begin{abstract}
ZIF-8@Polyacrylate carboxyl super-macroporous microspheres was synthesized by insitu growth method with the ZIF-8 nanoparticles loaded on the surface of PC-SMM for adsorptive removal of Malachite Green (MG) from water. The as-synthesized ZIF-8@PC-SMM was characterized by SEM and XRD experiments, and the adsorption kinetics were analyzed through the time-dependent adsorption results of MG on three adsorbents. The better adsorption performance of ZIF-8@PC-SMM for MG was exhibited compared with the PC-SMM, ZIF-8 and other adsorbents. The ZIF-8@PC-SMM obtained in this study have great potential for the removal of dyes or other containments in wastewater.
\end{abstract}

\section{Introduction}

Metal-organic frameworks (MOFs), an emerging class of micro-porous materials synthesized by metal ions clusters and organic linkers, have attracted great attention in gas storage, separation, catalysis, sensing, and drug delivery because of their high surface areas and uniformly structured nano-scale cavities [1-3]. Zeolitic imidazolate frameworks (ZIFs), a subclass of MOFs, are based on metal ions and imidazolate ligands [4]. Compared to other types of MOFs, ZIFs often exhibit unique properties of the microporosity, crystallinity, high surface area, and high chemical and thermal stability [4-8]. Recently, ZIFs has attracted considerable attention in diverse applications, including liquid adsorption [9,10], catalysis [11-13], and chemical analysis [14,15]. It has reported that ZIF-67 has been as an efficient heterogeneous catalyst for the cyclization reaction of 2-aminobenzoketones and benzylamine derivatives to form quinazoline products [13]. In addition, ZIF-67 was found to adsorptive remove phenol from aqueous system [16].

ZIF-8[Zn(2-methylimidazole $)_{2} \cdot 2 \mathrm{H}_{2} \mathrm{O}$ ], a kind of ZIFs, is composed of imidazolate organic ligands and $\mathrm{Zn}^{2+}$ center ions to form an octahedral cage structure. Cage diameter of the pore is about $1.2 \mathrm{~nm}$, pore diameter surface of the cage is approximately $0.34 \mathrm{~nm}$ [10]. Featured by Simple synthesis method, large accessible surface area and abundant active surface sites [10,17], ZIF-8 has attracted considerable attention in adsorption applications. Typically, ZIF-8 is taken as efficient and stable adsorbent materials and is widely used in removing dyes and small alcohols from water $[9,10]$.

Though ZIF-8 show good adsorption performance for dyes, the hydrophilicity, non-spherical morphologies and aggregated nanoparticles of ZIF-8 lead to the amounts of dye solution adsorbed on ZIF-8 are extremely low. So, enormous effort has been paid to form ZIF-8 composites by loading ZIF-8 on different support such as polymer [14], alumina [17], silica microspheres [18], in order to expand the applied range of ZIF-8. Surface functionality is critical for ZIF-8 growth in order to improve adherence and coverage on the support. Herein, we use the Polyacrylate carboxyl super-macroporous microspheres (PC-SMM) as support for the growth of ZIF-8 nanocrystals. The 
as-synthesized ZIF-8@PC-SMM has a microspherical shape similar to PC-SMM and also has the ZIF-8 properties. In this study, we characterized the structural properties of ZIF-8@PC-SMM, and investigated the adsorption performance of ZIF-8, PC-SMM and ZIF-8@PC-SMM for removal of Malachite Green (MG) from water.

\section{Experimental Section}

\section{Materials and Synthesis}

The solvents and chemicals used in this study are all commercially available and directly used without further purification. Typically, ZIF-8@PC-SMM was synthesized by the situ growth method as follow: $1.91 \mathrm{~g}$ cobalt nitrate hexahydrate $\left(\mathrm{Zn}\left(\mathrm{NO}_{3}\right)_{2} \cdot 6 \mathrm{H}_{2} \mathrm{O}\right)$ was dissolved in methanol $(50 \mathrm{ml})$ and water $(50 \mathrm{ml})$ in a $500-\mathrm{ml}$ beaker containing PC-SMM $(1.0 \mathrm{~g})$, stirring at $30^{\circ} \mathrm{C}, 150 \mathrm{rpm}$ of temperature oscillator for $24 \mathrm{~h}$ to allow the conjugation of $\mathrm{Zn}^{2+}$ to $-\mathrm{COO}^{-1}$. Then $100 \mathrm{ml}$ methanol solution of 2-methylimidazole $(4.9 \mathrm{~g})$ was added into the above mixed solution. The ZIF-8@PC-SMM products were collected by low speed centrifugation after reaction for $24 \mathrm{~h}$ and washed repeatedly with ethanol. The final products were dried at $80^{\circ} \mathrm{C}$ in the vacuum over for $24 \mathrm{~h}$ for late use. In addition, the synthesis conditions of ZIF-8 were the same as the synthesis of ZIF-8@PC-SMM, but not adding PC-SMM. ZIF-8 was collected by high speed centrifugation after reaction and washed repeatedly with ethanol. The ZIF-8 products were dried at $80^{\circ} \mathrm{C}$ in the vacuum oven for $24 \mathrm{~h}$ for late use.

\section{Characterization Experiments}

The morphologies of PC-SMM, ZIF-8 and ZIF-8@PC-SMM were observed by a scanning electron microscope (SEM, HITACHI SU8010). The crystalline structure of the PC-SMM, ZIF-8 and ZIF-8@PC-SMM samples were characterized by powder X-ray diffraction (XRD, Bruker D8 ADVANCE) with Cu-Ka and wavelength of $1.5418 \AA$ in the angle range of $5-50^{\circ}$.

\section{MG Adsorption Experiments}

In order to clearly to compare the adsorption performance of the PC-SMM, ZIF-8 and ZIF-8@PC-SMM for MG and study their kinetics. $10 \mathrm{~mL}$ MG aqueous solution with the initial concentration of $100 \mathrm{mg} \mathrm{L}-1$ were poured into $10 \mathrm{~mL}$ centrifugal tubes containing $10.0 \mathrm{mg}$ of PC-SMM, ZIF-8 and ZIF-8@PC-SMM samples, and then kept in a thermostatic incubator shaker (HZQ-X100) with a shaking speed of $150 \mathrm{rpm}$ at $30^{\circ} \mathrm{C}$ unless otherwise stated. After mixing different time (from 10 to $360 \mathrm{~min}$ ), the mixture solution was filtered with a $0.22 \mathrm{~mm}$ microporous membrane to collect the dilute solution and to measure the MG concentration by a UV-Vis spectrometer (UV759CRT, Shanghai) at the maximum absorbance of MG dye $(617 \mathrm{~nm})$ on the basis of Beer-Lamber law. The amount of adsorption at time $t, q_{t}\left(\mathrm{mg} \mathrm{g}^{-1}\right)$, was calculated by the Eq. 1 :

$$
q_{t}=\frac{\left(C_{0}-C_{t}\right) V}{m}
$$

where $C_{0}$ and $C_{t}\left(\mathrm{mg} \mathrm{L}^{-1}\right)$ are the liquid phase concentrations of $\mathrm{CR}$ at initial and any times, respectively; $V(\mathrm{~L})$ is the volume of the solution; $m(\mathrm{~g})$ is the mass of adsorbent used. When the equilibrium adsorption reached, the adsorption capacity at a nearly constant value is the equilibrium adsorption capacity $\left(q_{e}, \mathrm{mg} \mathrm{g}^{-1}\right)$.

\section{Results and Discussion}

\section{Characterization Results}

The sizes and morphologies of PC-SMM, ZIF-8 and ZIF-8@PC-SMM samples were examined by scanning electron microscope (SEM). The SEM images of PC-SMM in Fig. 1a and 1b showed a 
microspherical shape of PC-SMM. As shown in Fig. 1c, ZIF-8 nanoparticles have a good uniform particle size around $80 \mathrm{~nm}$ in diameter, well-defined facets, and sharp edges and corners. The ZIF-8 nanoparticles exhibited similar morphology to the ZIF-8 crystals as reported in literature [17]. Fig. 1d and 1e suggested that the porous surface of PC-SMM was covered by ZIF-8 nanoparticles, and the as-synthesized ZIF-8@PC-SMM kept the spherical shape after the PC-SMM modified through the insitu method.
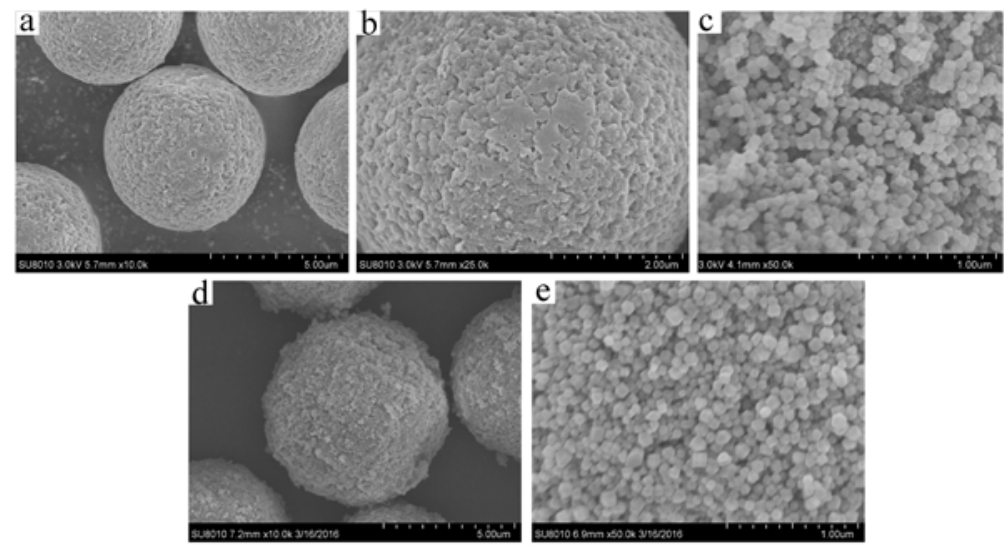

Fig. 1 SEM images of (a, b) PC-SMM, (c) ZIF-8 and (d, e) ZIF-8@PC-SMM.

The crystalline structure of the PC-SMM, ZIF-8and ZIF-8@PC-SMM samples were characterized by powder X-ray diffraction (XRD, Bruker D8 ADVANCE) with Cu-K $\alpha$ and wavelength of $1.5418 \AA$ in the angle range of $5-50^{\circ}$. Fig. 2 showed the XRD patterns of the PC-SMM, ZIF-8 and ZIF-8@PC-SMM samples. The XRD pattern of ZIF-8@PC-SMM is similar to that of PC-SMM, and also had some additional characteristic peaks matching the ZIF-8 crystalline structure. Furthermore, XRD pattern of ZIF-8@PC-SMM is well indexed to PC-SMM with good crystallization. Therefore, it indicated that ZIF-8 crystals were successfully loaded on the PC-SMM.

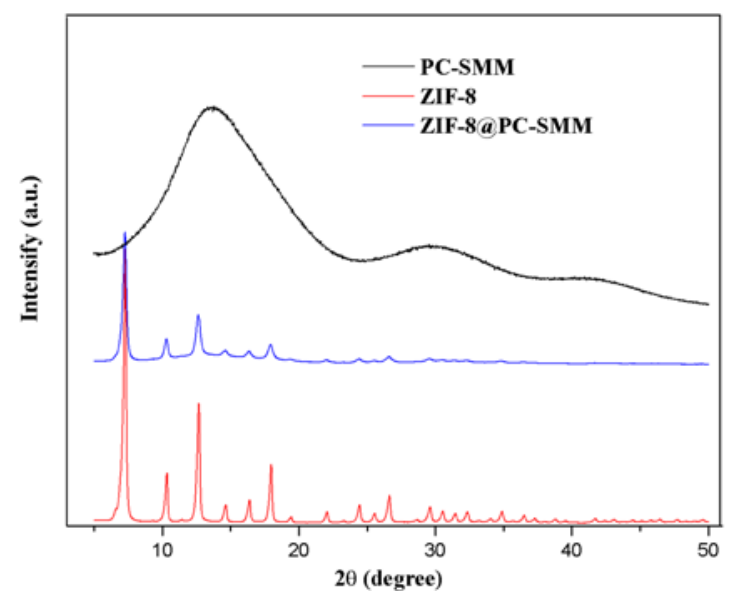

Fig. 2 XRD patterns of the ZIF-8, PC-SMM and ZIF-8@PC-SMM samples.

\section{Adsorption Results}

As mentioned above, the ZIF-8 nanoparticles growing on the surfaces of the PC-SMM leads to the ZIF-8@PC-SMM having ZIF-8 properties, which may play an important role in the adsorption of guest species. So ZIF-8@PC-SMM may have critical different adsorption behaviors with the PC-SMM. MG was used as a typical organic dye to evaluate the adsorption performances of the PC-SMM, ZIF-8 and ZIF-8@PC-SMM under the same conditions. The time-dependent adsorption of BBG on PC-SMM, ZIF-8 and ZIF-8@PC-SMM were investigated at the initial concentration of 100 $\mathrm{mg} \mathrm{L}^{-1}$ (as shown in Fig. 3) The adsorption capacity of PC-SMM, ZIF-8 and ZIF-8@PC-SMM for MG increased rapidly in the beginning ( $<60 \mathrm{~min})$ and then approached to equilibrium. In addition, the 
adsorption capacity was obviously in an order of PC-SMM < ZIF-8 < ZIF-8@PC-SMM in the whole adsorption process. The adsorption capacities of PC-SMM, ZIF-8 were 38.9 and $94.1 \mathrm{mg} \mathrm{g}^{-1}$, respectively. To our delight, the PC-SMM had the maximun adsorption capacity $\left(101.2 \mathrm{mg} \mathrm{g}^{-1}\right)$, which was about 2.6 and 1.1 times higher than the capacity of the PC-SMM and ZIF-8, respectively. Moreover, over 86\% of MG could be removed by ZIF-8@PC-SMM within the first 60 min. Therefore, these results revealing the rapid adsorption rate of MG on ZIF-8@PC-SMM and which do not interfere with the adsorption properties of ZIF-8.

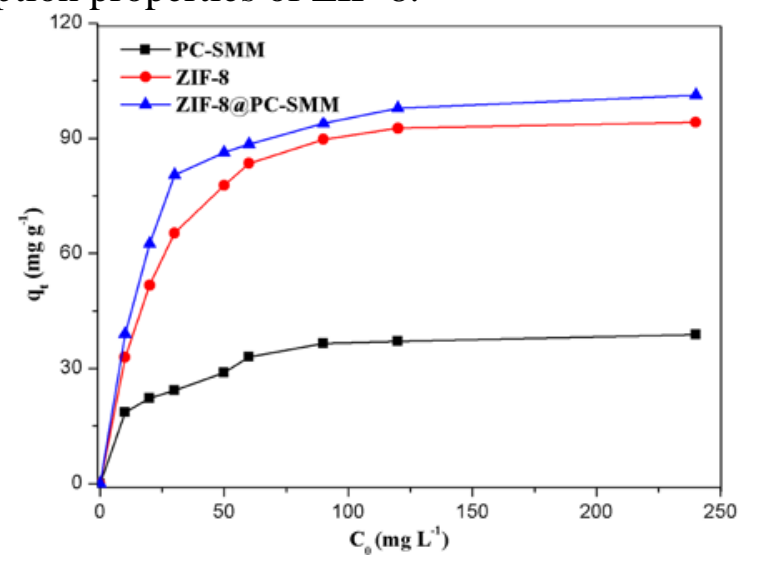

Fig. 3 Adsorption capacity of MG on PC-SMM, ZIF-8 and ZIF-8@PC-SMM at different time (initial concentration: $100 \mathrm{mg} \mathrm{L}^{-1}$, adsorption temperature: 30

To further gain insight into the adsorption kinetics, the quantitative kinetic order and adsorption rate constant for the adsorption of MG on three adsorbents were studied to model the kinetic data in Fig. 3. The pseudo-first-order and pseudo-second-order equations are typically described in the following two equations (Eq. 2 and Eq. 3), respectively:

$$
\begin{gathered}
\ln \left(q_{e}-q_{t}\right)=\ln q_{e}-k_{1} t \\
\frac{t}{q_{t}}=\frac{1}{k_{2} q_{e}^{2}}+\frac{t}{q_{e}}
\end{gathered}
$$

where $q_{t}$ and $q_{e}$ are the adsorption capacity $\left(\mathrm{mg} \mathrm{g}^{-1}\right)$ at a certain time $\mathrm{t}(\mathrm{min})$ and equilibrium,

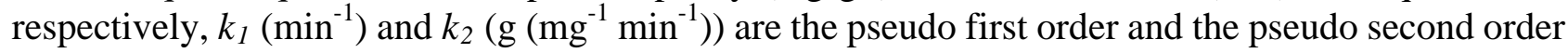
rate constants, respectively.

The fitting results of the adsorption kinetics for MG adsorption using different adsorbents at the initial concentration of $100 \mathrm{mg} \mathrm{L}^{-1}$ were shown in Fig. 4 (the $\ln \left(q_{e}-q_{t}\right)$ as a function of $t$ ) and Fig. 5 (the $t / q_{t}$ as a function of $t$ ). The kinetic parameters derived from the linear regressions of the fitting results were summarized in Table. 1 . It was clearly seen that the values of calculated adsorption capacities of PC-SMM, ZIF-8 and ZIF-8@PC-SMM respectively are 41.84, 102.04 and 107.53 mg $\mathrm{g}^{-1}$ for pseudo-second-order kinetic model, which were much closer to the experimental adsorption capacity. The higher regression coefficients $\left(R^{2}\right)$ and adsorption capacity suggested that the pseudo-second-order model is suitable for description of the MG adsorption process by three adsorbents. 

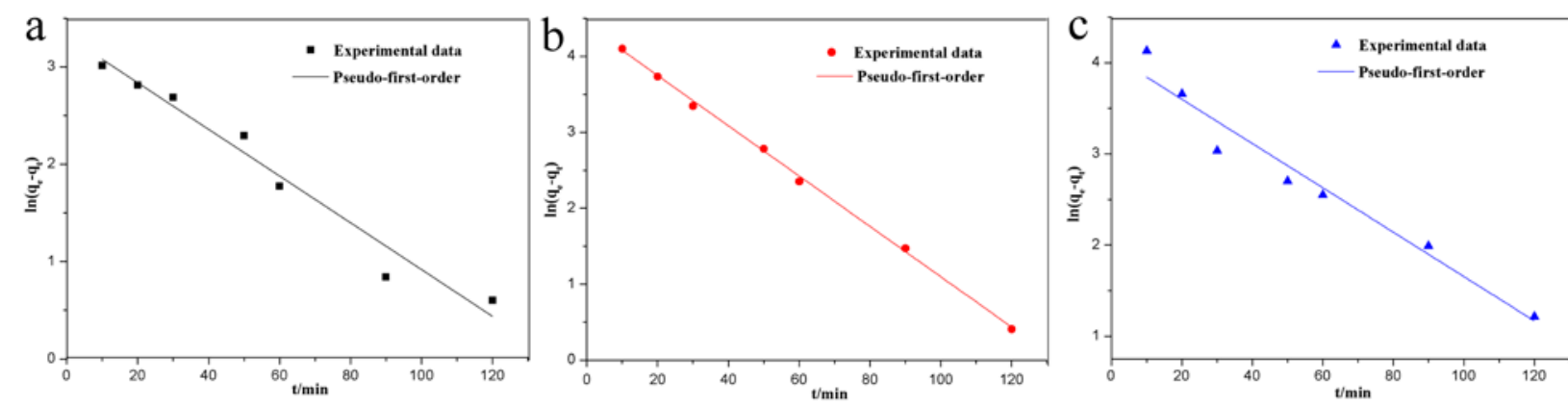

Fig. 4 The pseudo-first-order kinetics for adsorption of MG on the (a) PC-SMM; (b) ZIF-8 and (c) ZIF-8@PC-SMM (the adsorption time t from $20 \mathrm{~min}$ to $120 \mathrm{~min}$, and the $q_{t}$ at $240 \mathrm{~min}$ used as $q_{e}$ for fitting data).
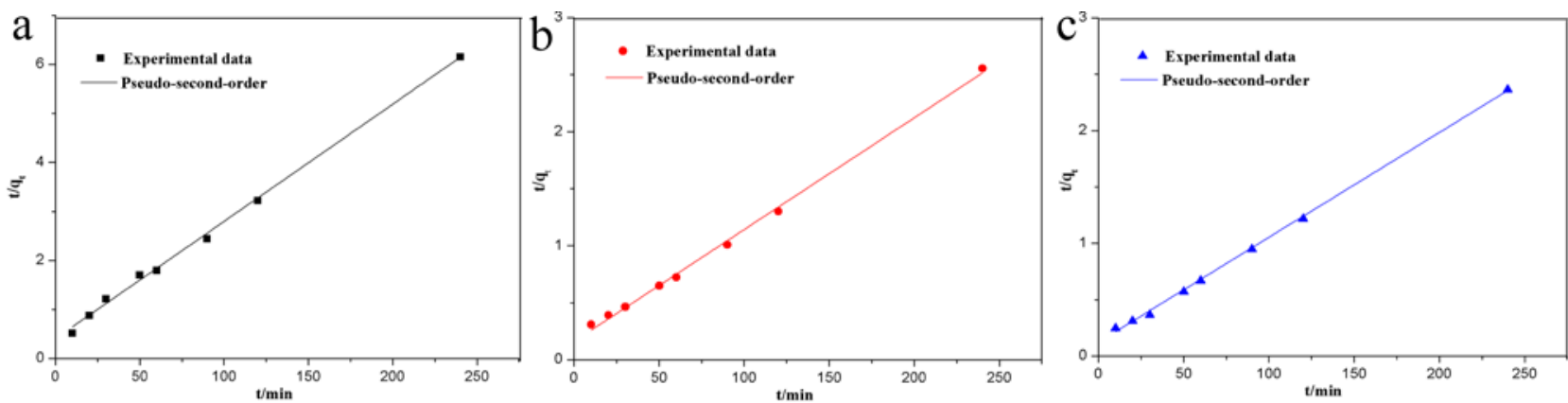

Fig. 5 The pseudo-second-order kinetics for adsorption of MG on the (a) PC-SMM; (b) ZIF-8 and (c) ZIF-8@PC-SMM (the adsorption time t from $20 \mathrm{~min}$ to $240 \mathrm{~min}$ ).

Table. 1 Kinetic parameters for MG adsorption on PC-SMM, ZIF-8 and ZIF-8@PC-SMM at $30{ }^{\circ} \mathrm{C}$.

\begin{tabular}{|c|c|c|c|c|c|c|}
\hline \multirow{2}{*}{ Adsorbents } & \multicolumn{3}{|c|}{ Pseudo-first-order kinetic model } & \multicolumn{2}{|c|}{ pseudo-second-order kinetic model } \\
\cline { 2 - 7 } & $\begin{array}{c}q_{e} \\
(\mathrm{mg} / \mathrm{g})\end{array}$ & $\begin{array}{c}k_{1} \\
\left(\mathrm{~min}^{-1}\right)\end{array}$ & $R^{2}$ & $\begin{array}{c}q_{e} \\
(\mathrm{mg} / \mathrm{g})\end{array}$ & $\begin{array}{c}k_{2} \\
(\mathrm{~g} /(\mathrm{mg} \mathrm{min}))\end{array}$ & $R^{2}$ \\
\hline PC-SMM & 27.21 & 0.0240 & 0.9679 & 41.84 & 0.0014 & 0.9977 \\
\hline ZIF-8 & 82.24 & 0.0331 & 0.9988 & 102.04 & 0.0006 & 0.9978 \\
\hline ZIF-8@PC-SMM & 58.79 & 0.0243 & 0.9593 & 107.53 & 0.0007 & 0.9991 \\
\hline
\end{tabular}

Moreover, ZIF-8@PC-SMM exhibited a capacity of $101.2 \mathrm{mg} \mathrm{g}^{-1}$ in adsorption time of $240 \mathrm{~min}$ which is fast and higher than many reported adsorption capacity (Table. 2), revealing that the ZIF-8@PC-SMM is a type of promising spherical adsorbents for MG removal from water. The ZIF-8@PC-SMM showing the fast and excellent adsorption may be caused by the strong electrostatic interactions between the MG and ZIF-8 loaded on the surface of PC-SMM and the $\pi-\pi$ interactions between the aromatic structures of MG and ZIF-8. In addition, the hydrophobicity of ZIF-8 easily leads agglomerate into large blocks, resulting part of the ZIF-8 nanoparticles could not be fully contact with dye molecules in short time. Therefore, the spherical shape of the ZIF-8@PC-SMM is helpful to make the ZIF-8 nanoparticles contact with MG, resulting in the increase of its adsorption capacity. 
Table2. Comparison of different adsorbents for MG adsorption capacities.

\begin{tabular}{|c|c|c|c|}
\hline Adsorbent & $\mathrm{T}\left({ }^{\circ} \mathrm{C}\right)$ & $\begin{array}{c}\text { Adsorption } \\
\text { capacity(mg/g) }\end{array}$ & Reference \\
\hline ZIF-8@PC-SMM & 30 & 101.2 & In this study \\
\hline Cyclodextrin-based material & 25 & 92 & 91 \\
\hline Alginate/ $\mathrm{Fe}_{3} \mathrm{O}_{4}$ composites & - & 50 & 20 \\
\hline Chitosan beads & 30 & 93 & 21 \\
\hline
\end{tabular}

\section{Conclusions}

In this study, we successfully synthesized the ZIF-8@PC-SMM with the ZIF-8 nanoparticles growing on the surface of PC-SMM by the insitu growth method. The combination of the spherical shape of PC-SMM and the microporous structure of enhanced the adsorption performance of ZIF-8@PC-SMM for MG. The maximun adsorption capacity of ZIF-8@PC-SMM for MG reached $101.2 \mathrm{mg} \mathrm{g}^{-1}$ at the initial concentration of $100 \mathrm{mg} \mathrm{L}^{-1}$, which was 2.6 and 1.1 times higher than on PC-SMM and pure ZIF-8. The efficient adsorption performance makes ZIF-8@PC-SMM promising as novel adsorbent for the adsorption of MG and other dyes from wastewater.

\section{Acknowledgement}

This research was financially supported by the Fundamental Research Funds for the Central Universities (NO. 2017JC03), National Natural Science Foundation of China (No. 21406013 and No. 21576029), and Beijing Natural Science Foundation (No. 2154054), and Scientic Research Foundation for the Returned Overseas Chinese Scholars, State Education Ministry (No. 14JLX-03).

\section{References}

[1] H. Furukawa, K. E. Cordova, M. O’Keeffe, et al. The chemistry and applications of metal-organic frameworks[J]. Science, 2013, 341(6149): 1230444.

[2] S. Qiu, M. Xue, G. Zhu. Metal-organic framework membranes: from synthesis to separation application[J]. Chemical Society Reviews, 2014, 43(16): 6116-6140.

[3] Y. Chen, S. Li, X. Pei, et al. A Solvent-Free Hot-Pressing Method for Preparing Metal-Organic-Framework Coatings[J]. Angewandte Chemie International Edition, 2016, 55(10): 3419-3423.

[4] K. S. Park, Z. Ni, A. P. Côté, et al. Exceptional chemical and thermal stability of zeolitic imidazolate frameworks[J]. Proceedings of the National Academy of Sciences, 2006, 103(27): 10186-10191.

[5] D. Bradshaw, A. Garai, J. Huo. Metal-organic framework growth at functional interfaces: thin films and composites for diverse applications[J]. Chemical Society Reviews, 2012, 41(6): 2344-2381.

[6] A. Ahmed, M. Forster, J. Jin, et al. Tuning morphology of nanostructured ZIF-8 on silica microspheres and applications in liquid chromatography and dye degradation[J]. ACS applied materials \& interfaces, 2015, 7(32): 18054-18063.

[7] R. Banerjee, A. Phan, B. Wang, et al. High-throughput synthesis of zeolitic imidazolate frameworks and application to CO2 capture[J]. Science, 2008, 319(5865): 939-943.

[8] A. Phan, C. J. Doonan, F. J. Uribe-Romo, et al. Synthesis, structure, and carbon dioxide capture properties of zeolitic imidazolate frameworks[J]. Acc. Chem. Res, 2010, 43(1): 58-67. 
[9] J. Q. Jiang, C. X. Yang, X. P. Yan. Zeolitic imidazolate framework-8 for fast adsorption and removal of benzotriazoles from aqueous solution[J]. ACS applied materials \& interfaces, 2013, 5(19): 9837-9842.

[10] J. Li, Y. Wu, Z. Li, et al. Zeolitic imidazolate framework-8 with high efficiency in trace arsenate adsorption and removal from water[J]. The Journal of Physical Chemistry C, 2014, 118(47): 27382-27387.

[11] A. Corma, H. García, F. X. Llabrés i Xamena. Engineering metal organic frameworks for heterogeneous catalysis[J]. Chemical reviews, 2010, 110(8): 4606-4655.

[12] L. Yang, L. Yu, G. Diao, et al. Zeolitic imidazolate framework-68 as an efficient heterogeneous catalyst for chemical fixation of carbon dioxide[J]. Journal of Molecular Catalysis A: Chemical, 2014, 392: 278-283.

[13] Truong T, Hoang T M, Nguyen C K, et al. Expanding applications of zeolite imidazolate frameworks in catalysis: synthesis of quinazolines using ZIF-67 as an efficient heterogeneous catalyst[J]. RSC Advances, 2015, 5(31): 24769-24776.

[14] X. Cao, L. Dai, J. Liu, et al. Fabrication of ZIF-8@ super-macroporous poly (glycidyl methacrylate) microspheres[J]. Inorganic Chemistry Communications, 2014, 50: 65-69.

[15] A. Centrone, E. E. Santiso, T. A. Hatton. Separation of chemical reaction intermediates by metal-organic frameworks[J]. Small, 2011, 7(16): 2356-2364.

[16] Y. Pan, Z. Li, Z. Zhang, et al. Adsorptive removal of phenol from aqueous solution with zeolitic imidazolate framework-67[J]. Journal of environmental management, 2016, 169: 167-173.

[17] Y. Pan, Z. Lai. Sharp separation of C2/C3 hydrocarbon mixtures by zeolitic imidazolate framework-8 (ZIF-8) membranes synthesized in aqueous solutions[J]. Chemical Communications, 2011, 47(37): 10275-10277.

[18] A. Ahmed, M. Forster, J. Jin, et al. Tuning morphology of nanostructured ZIF-8 on silica microspheres and applications in liquid chromatography and dye degradation[J]. ACS applied materials \& interfaces, 2015, 7(32): 18054-18063.

[19] G. Crini, H. N. Peindy, F. Gimbert, et al. Removal of CI Basic Green 4 (Malachite Green) from aqueous solutions by adsorption using cyclodextrin-based adsorbent: Kinetic and equilibrium studies[J]. Separation and Purification Technology, 2007, 53(1): 97-110.

[20] A. Mohammadi, H. Daemi and M. Barikani. Fast removal of malachite green dye using novel superparamagnetic sodium alginate-coated $\mathrm{Fe}_{3} \mathrm{O}_{4}$ nanoparticles[J]. International journal of biological macromolecules, 2014, 69: 447-455.

[21] Z. Bekçi, C. Özveri, Y. Seki, et al. Sorption of malachite green on chitosan bead[J]. Journal of hazardous materials, 2008, 154(1): 254-261. 